\title{
Bounds on Certain Multiplications of Affine Combinations
}

\author{
Joan Boyar* \\ Loyola University Chicago \\ Odense University \\ Faith Fich ${ }^{\dagger}$ \\ University of Toronto \\ Kim S. Larsen ${ }^{\ddagger}$ \\ Aarhus University
}

August 1992

\begin{abstract}
Let $A$ and $B$ be $n \times n$ matrices the entries of which are affine combinations of the variables $a_{1}, \ldots, a_{m}, b_{1}, \ldots, b_{m}$ over $\mathrm{GF}(2)$. Suppose that, for each $i, 1 \leq i \leq m$, the term $a_{i} b_{i}$ is an element of the product matrix $C=A \cdot B$. What is the maximum value that $m$ can have as a function of $n$ ? This question arises from a recent technique for improving the communication complexity of zero-knowledge proofs.

The obvious upper bound of $n^{2}$ is improved to $n^{2} / \sqrt[3]{3}+O(n)$. Tighter bounds are obtained for smaller values of $n$. The bounds for $n=2, n=3$, and $n=4$ are tight.
\end{abstract}

*Supported in part by NSA Grant Number MDA90-H-4016. Some of this work was done while visiting the University of Toronto and Aarhus University.

†'Supported in part by Natural Science and Engineering Research Council of Canada grant A-9176 and the Information Technology Research Centre of Ontario

${ }^{\ddagger}$ Some of this work was done while visiting the University of Toronto. 


\section{Introduction}

The problem described in the abstract and discussed in this paper is motivated by recent results in cryptography. A new technique for improving the communication complexity of zero-knowledge proofs for circuit satisfiability was presented in [BBP91]. The key idea is that the Prover shows that all the inputs and outputs to the AND gates are correct by showing that a matrix multiplication is correct. Suppose that the inputs to $m$ AND gates are $\left(a_{1}, b_{1}\right),\left(a_{2}, b_{2}\right), \ldots,\left(a_{m}, b_{m}\right)$, and that the outputs are $c_{1}, c_{2}, \ldots, c_{m}$, respectively. Given encryptions for the $a_{i}{ }^{\prime} \mathrm{s}, b_{i}{ }^{\prime} \mathrm{s}$, and $c_{i}{ }^{\prime} \mathrm{s}$, the Prover is trying to show that the following equalities hold in $\operatorname{GF}(2)$ : $a_{1} b_{1}=c_{1}, a_{2} b_{2}=c_{2}, \ldots, a_{m} b_{m}=c_{m}$. The variables $a_{1}, a_{2}, \ldots, a_{m}$ are put in an $n \times n$ matrix $A$ which has zeros as its remaining elements. The variables $b_{1}, b_{2}, \ldots, b_{m}$ are put in an $n \times n$ matrix $B$ which also has zeros as its remaining elements. These variables and zeros are placed so that every one of the $c_{i}$ 's is contained somewhere in the product matrix $C=A \cdot B$. For example, if the $a_{i}$ 's and the $b_{i}$ 's are on the diagonals of their respective matrices, and if the other entries of these matrices are 0 , the $c_{i}$ 's will be on the diagonal of the product. The usefulness of the technique in [BBP91], however, depends on $m$ being significantly larger than $n$; the larger, the better.

The smallest interesting example has $m=6$ and $n=3$ :

$$
\left(\begin{array}{ccc}
a_{1} & a_{2} & 0 \\
a_{3} & 0 & a_{4} \\
0 & a_{5} & a_{6}
\end{array}\right) \cdot\left(\begin{array}{ccc}
b_{3} & b_{1} & 0 \\
b_{5} & 0 & b_{2} \\
0 & b_{6} & b_{4}
\end{array}\right)=\left(\begin{array}{ccc}
a_{1} b_{3}+a_{2} b_{5} & a_{1} b_{1} & a_{2} b_{2} \\
a_{3} b_{3} & a_{3} b_{1}+a_{4} b_{6} & a_{4} b_{4} \\
a_{5} b_{5} & a_{6} b_{6} & a_{5} b_{2}+a_{6} b_{4}
\end{array}\right) .
$$

A construction in [BBP91] gives the values $m=32^{t}$ and $n=8^{t}$ for any positive integer $t$. Thus, it is possible to put $m=n^{5 / 3}$ entries in an $n \times n$ matrix if $n$ is a power of 8. Although this is the best known result in the practical range, an asymptotic improvement of theoretical interest, also described in [BBP91], has been discovered by Szemerédi [Sze], using a result of [SS42]. It is possible to put $m$ entries in matrices of size $n \times n$, where $n \leq(\sqrt{m})^{1+\varepsilon_{m}}$ and $\varepsilon_{m}=4 \sqrt{2} / \sqrt{\lg m}$, which is better than the other construction, provided that $m \geq 2^{128}$. Since $\varepsilon_{m}$ approaches zero as $m$ approaches infinity, $m$ is nearly linear in $n^{2}$, the number of entries in the matrix.

In all these examples, the matrix $A$ contains only $a_{i}$ 's and zeros and the matrix $B$ contains only $b_{i}$ 's and zeros. This restriction is neither stated nor 
necessary for the technique described in [BBP91]. In fact, because of various properties of the encryption scheme used, the entries in both $A$ and $B$ could also have the form $\sum_{j=1}^{k} x_{j}$ where each $x_{j} \in\left\{a_{1}, a_{2}, \ldots, a_{m}, b_{1}, b_{2}, \ldots, b_{m}, 1\right\}$. Thus, these entries can be affine combinations of the variables. For example, in $2 \times 2$ matrices, one could have:

$$
\left(\begin{array}{cc}
a_{1}+a_{2}+b_{1}+1 & b_{1} \\
a_{1}+a_{2} & a_{1}
\end{array}\right) \cdot\left(\begin{array}{cc}
b_{1} & b_{2} \\
a_{2} & b_{2}
\end{array}\right)=\left(\begin{array}{cc}
a_{1} b_{1} & a_{1} b_{2}+a_{2} b_{2}+b_{2} \\
a_{1} b_{1}+a_{2} b_{1}+a_{1} a_{2} & a_{2} b_{2}
\end{array}\right)
$$

This example just gives $m=n=2$, which is no improvement over what can be done without using affine combinations In fact, there are no known examples where removing the original restrictions does give any improvement.

For a given $n$, let $M(n)$ denote the maximumvalue that $m$ can have. Then $M(n) \leq n^{2}$. In this paper, we improve this bound to $n^{2} / \sqrt[3]{3}+O(n)$. This bound is definitely not tight for small $n$. We prove other results which give tighter bounds when $n$ is small, and exact bounds for $n=2, n=3$, and $n=4$.

\section{Asymptotic Bounds}

Given a matrix $C$, choose $k \geq 2$ rows and $\lfloor n / k\rfloor+1$ columns and consider the $k \times(\lfloor n / k\rfloor+1)$ submatrix of $C$ consisting of the intersection of these rows and columns. In this section, we show that no such submatrix can consist entirely of distinct $c_{i}$ 's and use this fact to obtain an upper bound on $M(n)$.

In order to prove this, we use a result from the study of straight-line programs over fields. These are programs in which the $i$ th statement has the form $V_{i} \leftarrow U_{j}$ or the form $V_{i} \leftarrow U_{j} \odot U_{k}$, where each of $U_{j}$ and $U_{k}$ is either an input to the program, some variable $V_{l}$ with $l<i$, or a field constant, and $\odot$ is addition or multiplication. The next lemma follows from results of [Win70]. The proof given here is more direct and it is included for the sake of completeness.

Lemma 1 Let $a_{1}, a_{2}, \ldots, a_{k}$ and $b_{1}, b_{2}, \ldots, b_{k}$ be independent variables over GF(2). Then any straight-line program for computing the inner product $\sum_{i=1}^{k} a_{i} b_{i}$ requires at least $k$ (nonscalar) multiplications.

Proof Suppose the claim is false. Consider the smallest value $k$ for which 
there is a straight-line program $P$ computing the inner product $\sum_{i=1}^{k} a_{i} b_{i}$ using less than $k$ multiplications. Since even the product $a_{1} b_{1}$ cannot be computed without any multiplications, $P$ must contain at least one (nonscalar) multiplication. Consider the first statement $z \leftarrow x \cdot y$ in $P$ that involves a multiplication. Both $x$ and $y$ are affine combinations of one or more of the variables. Without loss of generality, say $x=a_{k}+x^{\prime}$ where $x^{\prime}$ is a constant or an affine combination of other variables.

Construct a straight-line program $P^{\prime}$ from $P$ by prepending the statements $b_{k} \leftarrow 0$ and $a_{k} \leftarrow x^{\prime}$ and replacing the statement $z \leftarrow x \cdot y$ by $z \leftarrow 0$. Then $P^{\prime}$ computes $\sum_{i=1}^{k-1} a_{i} b_{i}$. None of the new statements in $P^{\prime}$ involve any multiplications, so $P^{\prime}$ uses fewer than $k-1$ multiplications. This contradicts the minimality of $k$.

Lemma 2 Let $a_{1}, a_{2}, \ldots, a_{m}$ and $b_{1}, b_{2}, \ldots, b_{m}$ be distinct variables over $G F(2)$, and suppose $c_{i}=a_{i} b_{i}$ for $1 \leq i \leq m$. Let $A, B$, and $C$ be $n \times n$ matrices such that $A \cdot B=C$. Suppose that the entries of $A$ and $B$ are affine combinations of the variables. If there exists an $s \times t$ submatrix of $C$ in which every element is distinct and is one of the $c_{i}$ 's, then st $\leq n$. Furthermore, if $s t=n$, then no other element in any of those $s$ rows or $t$ columns of $C$ is a different $c_{i}$.

Proof Consider an $s \times t$ submatrix of $C$ consisting of the intersection of rows $r_{1}, r_{2}, \ldots, r_{s}$ and columns $q_{1}, q_{2}, \ldots, q_{t}$ and which contains the entries $c_{1}, \ldots, c_{s t}$. Since $C=A \cdot B$,

$$
\begin{aligned}
\sum_{i=1}^{s \times t} a_{i} b_{i}=\sum_{i=1}^{s \times t} c_{i} & =\sum_{j=1}^{s} \sum_{l=1}^{t} C\left[r_{j}, q_{l}\right] \\
& =\sum_{j=1}^{s} \sum_{l=1}^{t} \sum_{k=1}^{n} A\left[r_{j}, k\right] \cdot B\left[k, q_{l}\right] \\
& =\sum_{j=1}^{s} \sum_{k=1}^{n} A\left[r_{j}, k\right] \cdot\left(\sum_{l=1}^{t} B\left[k, q_{l}\right]\right) \\
& =\sum_{k=1}^{n}\left(\sum_{j=1}^{s} A\left[r_{j}, k\right]\right) \cdot\left(\sum_{l=1}^{t} B\left[k, q_{l}\right]\right) .
\end{aligned}
$$

Each of the terms $A\left[r_{j}, k\right]$ and $B\left[k, q_{l}\right]$ is an affine combination of the $a_{i}$ 's and $b_{i}$ 's, so the sums $\sum_{j=1}^{s} A\left[r_{j}, k\right]$ and $\sum_{l=1}^{t} B\left[k, q_{l}\right]$ can be computed without any 
multiplications. Thus the right hand side can be computed by a straight-line program with only $n$ multiplications. By lemma 1 , the left hand side requires at least st multiplications. Thus, st $\leq n$.

Now assume $s t=n$. Then, for each $k \in\{1, \ldots, n\}, \sum_{j=1}^{s} A\left[r_{j}, k\right]$ is an affine combination of the variables $a_{1}, \ldots, a_{n}, b_{1}, \ldots, b_{n}$. To see why, suppose $\sum_{j=1}^{s} A\left[r_{j}, k^{\prime}\right]=a_{n+1}+d$, where $k^{\prime} \in\{1, \ldots, n\}$ and $d$ is an affine combination of variables excluding $a_{n+1}$. Let $A^{\prime}, B^{\prime}$, and $C^{\prime}$ be the matrices obtained by replacing all occurrences of $a_{n+1}$ by $d$ in $A, B$, and $C$, respectively. Then $A^{\prime} \cdot B^{\prime}=C^{\prime}$. Furthermore, since $C\left[r_{j}, q_{l}\right]$ does not contain $a_{n+1}, C^{\prime}\left[r_{j}, q_{l}\right]=$ $C\left[r_{j}, q_{l}\right]$ for all $j \in\{1, \ldots, s\}, l \in\{1, \ldots, t\}$. Thus

$$
\begin{aligned}
\sum_{i=1}^{s \times t} a_{i} b_{i} & =\sum_{j=1}^{s} \sum_{l=1}^{t} C\left[r_{j}, q_{l}\right] \\
& =\sum_{j=1}^{s} \sum_{l=1}^{t} C^{\prime}\left[r_{j}, q_{l}\right] \\
& =\sum_{k=1}^{n}\left(\sum_{j=1}^{s} A^{\prime}\left[r_{j}, k\right]\right) \cdot\left(\sum_{l=1}^{t} B\left[k, q_{l}\right]\right) .
\end{aligned}
$$

Since $\sum_{j=1}^{s} A\left[r_{j}, k^{\prime}\right]=a_{n+1}+d$, it follows that $\sum_{j=1}^{s} A^{\prime}\left[r_{j}, k^{\prime}\right]=0$. But this implies that the right hand side can be computed by a straight-line program using only $n-1$ multiplications, contradicting lemma 1 .

Similarly, $\sum_{l=1}^{t} B\left[k, q_{l}\right]$ is an affine combination of $a_{1}, \ldots, a_{n}, b_{1}, \ldots, b_{n}$, for each $k \in\{1, \ldots, n\}$.

In fact, for each $j \in\{1, \ldots, s\}$ and $k \in\{1, \ldots, n\}, A\left[r_{j}, k\right]$ is, itself, an affine combination of the variables $a_{1}, \ldots, a_{n}, b_{1}, \ldots, b_{n}$. Suppose, to the contrary, that $A\left[r, k^{\prime}\right]=a_{n+1}+d$, where $\left\{r \in r_{1}, \ldots, r_{s}\right\}, k^{\prime} \in\{1, \ldots, n\}$, and $d$ is an affine combination of variables excluding $a_{n+1}$. Let $e=\sum_{j=1}^{s} A\left[r_{j}, k^{\prime}\right]$ and let $A^{\prime}$ and $A^{\prime \prime}$ be obtained by replacing all occurrences of $a_{n+1}$ in $A$ by 0 and $e$, respectively. Define $B^{\prime}, B^{\prime \prime}, C^{\prime}$, and $C^{\prime \prime}$ analogously. Then $A^{\prime} \cdot B^{\prime}=C^{\prime}$ and $A^{\prime \prime} \cdot B^{\prime \prime}=C^{\prime \prime}$. Since $\sum_{j=1}^{s} A\left[r_{j}, k\right]$ and $\sum_{l=1}^{t} B\left[k, q_{l}\right]$ are not functions of $a_{n+1}$, for any $k \in\{1, \ldots, n\}$, and $C\left[r_{i}, q_{l}\right]$ is not a function of $a_{n+1}$, for any $j \in\{1, \ldots, s\}$ and $l \in\{1, \ldots, t\}$, 


$$
\begin{aligned}
& \sum_{j=1}^{s} A\left[r_{j}, k\right]=\sum_{j=1}^{s} A^{\prime}\left[r_{j}, k\right]=\sum_{j=1}^{s} A^{\prime \prime}\left[r_{j}, k\right], \\
& \sum_{l=1}^{t} B\left[k, q_{l}\right]=\sum_{l=1}^{t} B^{\prime}\left[k, q_{l}\right]=\sum_{l=1}^{t} B^{\prime \prime}\left[k, q_{l}\right], \text { and } \\
& C\left[r_{j}, q_{l}\right]=C^{\prime}\left[r_{j}, q_{l}\right]=C^{\prime \prime}\left[r_{j}, q_{l}\right] .
\end{aligned}
$$

Thus,

$$
\begin{aligned}
\sum_{l=1}^{t} \sum_{k=1}^{n} A^{\prime}[r, k] \cdot B^{\prime}\left[k, q_{l}\right] & =\sum_{l=1}^{t} C^{\prime}\left[r, q_{l}\right] \\
& =\sum_{l=1}^{t} C^{\prime \prime}\left[r, q_{l}\right] \\
& =\sum_{l=1}^{t} \sum_{k=1}^{n} A^{\prime \prime}[r, k] \cdot B^{\prime \prime}\left[k, q_{l}\right] \\
& =\sum_{k=1}^{n} A^{\prime \prime}[r, k] \cdot\left(\sum_{l=1}^{t} B^{\prime \prime}\left[k, q_{l}\right]\right) \\
& =\sum_{k=1}^{n} A^{\prime \prime}[r, k] \cdot\left(\sum_{l=1}^{t} B^{\prime}\left[k, q_{l}\right]\right) \\
& =\sum_{l=1}^{t} \sum_{k=1}^{n} A^{\prime \prime}[r, k] \cdot B^{\prime}\left[k, q_{l}\right] \\
\text { and } A^{\prime \prime}\left[r, k^{\prime}\right]+\sum_{\substack{j=1 \\
r_{j} \neq r}}^{s} A^{\prime}\left[r_{j}, k^{\prime}\right] & =A^{\prime \prime}\left[r, k^{\prime}\right]+A^{\prime}\left[r, k^{\prime}\right]+\sum_{j=1}^{s} A^{\prime}\left[r_{j}, k^{\prime}\right] \\
& =A^{\prime \prime}\left[r, k^{\prime}\right]+A^{\prime}\left[r, k^{\prime}\right]+\sum_{j=1}^{s} A\left[r_{j}, k^{\prime}\right] \\
& =(e+d)+d+e=0 .
\end{aligned}
$$

From these facts, it follows that

$$
\begin{aligned}
\sum_{i=1}^{s \times t} a_{i} b_{i} & =\sum_{j=1}^{s} \sum_{l=1}^{t} \sum_{k=1}^{n} A^{\prime}\left[r_{j}, k\right] \cdot B^{\prime}\left[k, q_{l}\right] \\
& =\sum_{l=1}^{t} \sum_{k=1}^{n} A^{\prime}[r, k] \cdot B^{\prime}\left[k, q_{l}\right]+\sum_{\substack{j=1 \\
r_{j} \neq r}}^{s} \sum_{l=1}^{t} \sum_{k=1}^{n} A^{\prime}\left[r_{j}, k\right] \cdot B^{\prime}\left[k, q_{l}\right]
\end{aligned}
$$




$$
\begin{aligned}
& =\sum_{l=1}^{t} \sum_{k=1}^{n} A^{\prime \prime}[r, k] \cdot B^{\prime}\left[k, q_{l}\right]+\sum_{\substack{j=1 \\
r_{j} \neq r}}^{s} \sum_{l=1}^{t} \sum_{k=1}^{n} A^{\prime}\left[r_{j}, k\right] \cdot B^{\prime}\left[k, q_{l}\right] \\
& =\sum_{k=1}^{n}\left(\begin{array}{c}
\left.A^{\prime \prime}[r, k]+\sum_{\substack{j=1 \\
r_{j} \neq r}}^{s} A^{\prime}\left[r_{j}, k\right]\right) \cdot\left(\sum_{l=1}^{t} B^{\prime}\left[k, q_{l}\right]\right) \\
=\sum_{\substack{k=1 \\
k \neq k^{\prime}}}^{n}\left(A^{\prime \prime}[r, k]+\sum_{\substack{j=1 \\
r_{j} \neq r}}^{s} A^{\prime}\left[r_{j}, k\right]\right) \cdot\left(\sum_{l=1}^{t} B^{\prime}\left[k, q_{l}\right]\right)
\end{array}\right)
\end{aligned}
$$

But this contradicts lemma 1, since the right hand side can be computed by a straight-line program using only $n-1$ multiplications.

Similarly, for each $l \in\{1, \ldots, t\}$ and $k \in\{1, \ldots, n\}, B\left[k, q_{l}\right]$ is an affine combination of the variables $a_{1}, \ldots, a_{n}, b_{1}, \ldots, b_{n}$.

If $a_{n+1} b_{n+1}=C[r, q]=\sum_{k=1}^{n} A[r, k] \cdot B[k, q]$, then a $k \in\{1, \ldots, n\}$ exists such that $a_{n+1}$ is contained in $A[r, k]$ and $b_{n+1}$ is contained in $B[k, q]$, or vice versa. This implies that $r \notin\left\{r_{1}, \ldots, r_{s}\right\}$ and $q \notin\left\{q_{1}, \ldots, q_{l}\right\}$.

Given an $n \times n$ matrix $C$ with $\mathrm{m}$ distinct $c_{i}$ 's, construct an $n \times n$ matrix $D$ with $m$ ones (corresponding to distinct $c_{i}$ 's) and $n^{2}-m$ zeros. If $C$ is the product of two matrices the entries of which are affine combinations of the variables $a_{1}, \ldots, a_{m}, b_{1}, \ldots, b_{m}$, we say that the zero-one matrix $D$ is a representative matrix.

Corollary 1 If an $n \times n$ representative matrix has an $s \times t$ submatrix containing only ones, then st $\leq n$. Furthermore, if st $=n$, then no other element in any of those $s$ rows or $t$ columns is one.

To prove an upper bound on $M(n)$, it suffices to prove an upper bound on the maximum number of ones in any $n \times n$ representative matrix. This is a special case of the problem: determine the least positive integer $k_{i, j}(m, n)$ such that if a zero-one matrix of size $m \times n$ contains $k_{i, j}(m, n)$ ones, then it must have an $i \times j$ submatrix containing only ones. This is a generalization of 
a problem originally posed by Zarankiewicz [Zar51]. The first upper bound on this problem,

$$
k_{i, j}(m, n) \leq 1+(i-1) n+\left\lfloor(j-1)^{1 / i} n^{1-1 / i} m\right\rfloor,
$$

was given by Hyltén-Cavallius [HC58], using the methods of Kövari, Sós, and Turán [KST54]. This has been improved slightly by others, including Guy and Znám [GZ91] and Roman [Rom75]. Tighter results have been found for small values of $i$ and $j$. In particular, Hyltén-Cavallius [HC58] has shown that

$$
k_{2, j}(m, n) \leq 1+\left\lfloor\frac{1}{2} n+\sqrt{(j-1) n m(m-1)+\frac{1}{4} n^{2}}\right\rfloor .
$$

All of these upper bounds are obtained using Dirichlet's pigeonhole principle as the main tool, and we use the same techniques in lemma 5.

The following lemmas give upper bounds on the number of ones in an $n \times n$ representative matrix and thus upper bounds on $M(n)$.

Lemma 3 Suppose that an $n \times n$ representative matrix $D$ contains more than $(1-1 / k) n^{2}-(k-2) n$ ones. Then it contains no $k \times\lceil n / k\rceil$ submatrix consisting entirely of ones.

Proof If $n$ is not divisible by $k$, then $k\lceil n / k\rceil>n$, so, by corollary $1, D$ does not contain a $k \times\lceil n / k\rceil$ submatrix consisting entirely of ones.

Therefore suppose that $n$ is divisible by $k$ and $D$ contains a $k \times n / k$ submatrix consisting entirely of ones. Then, by corollary 1 , none of the $k(n-n / k)$ other elements in the same rows and none of the $(n-k) n / k$ other elements in the same columns are ones. Hence $D$ contains at most $n^{2}-n k+n-n^{2} / k+n=$ $(1-1 / k) n^{2}-(k-2) n$ ones.

Lemma 4 Suppose $D$ is an $n \times n$ representative matrix, $n \geq 2$. Then $D$ contains at most $\frac{n}{2}(1+\sqrt{1+4(\lceil n / 2\rceil-1)(n-1)})=n^{2} / \sqrt{2}+O(n)$ ones.

Proof By lemma 3, we may assume that $D$ does not contain a $2 \times\lceil n / 2\rceil$ submatrix consisting entirely of ones. Thus, we can apply the result of Hyltén-Cavallius [HC58] on $k_{2, j}(m, n)$, setting $j=\lceil n / 2\rceil$ and $m=n$. Since $k_{2,\lceil n / 2\rceil}(n, n)$ is the number of ones necessary to ensure that a $2 \times\lceil n / 2\rceil$ submatrix containing only ones exists, the value we need is one less. 
This result implies that $M(2) \leq 2, M(3) \leq 6$, and $M(4) \leq 9$. The lower bounds, $M(2) \geq 2$ and $M 3 \geq 6$, follow from the examples in the introduction. The following example, in which each $*$ represents some uninteresting bilinear form, gives that $M(4) \geq 9$.

$$
\left(\begin{array}{cccc}
a_{1} & a_{2} & a_{3} & 0 \\
0 & a_{4} & 0 & a_{5} \\
a_{6} & 0 & 0 & a_{7} \\
0 & 0 & a_{8} & a_{9}
\end{array}\right) \cdot\left(\begin{array}{cccc}
b_{1} & 0 & 0 & b_{6} \\
0 & b_{2} & 0 & b_{4} \\
0 & 0 & b_{3} & b_{8} \\
b_{9} & b_{7} & b_{5} & 0
\end{array}\right)=\left(\begin{array}{cccc}
a_{1} b_{1} & a_{2} b_{2} & a_{3} b_{3} & * \\
* & * & a_{5} b_{5} & a_{4} b_{4} \\
* & a_{7} b_{7} & * & a_{6} b_{6} \\
a_{9} b_{9} & * & * & a_{8} b_{8}
\end{array}\right) .
$$

Thus $M(2)=2, M(3)=6$, and $M(4)=9$.

The proof of lemma 4 only used corollary 1 for $s=2$. The same technique can also be applied for other values of $s$. Using the standard pigeonhole technique, the value $s=3$ gives the best result asymptotically. The results of [HC58], [GZ91], and [Rom75] all give the asymptotic result we obtain in the following lemma, but since our problem is less general, the result given here is slightly tighter.

Lemma 5 Suppose $D$ is an $n \times n$ representative matrix, $n \geq 4$. Let

$$
u=\frac{1}{2}(\lceil n / 3\rceil-1)(n-1)(n-2) \text { and } v=\sqrt{u^{2}-1 / 27} .
$$

Then $D$ contains at most

$$
n(1+\sqrt[3]{u+v}+\sqrt[3]{u-v})=n^{2} / \sqrt[3]{3}+O(n)
$$

ones.

Proof By lemma 3, we may assume that $D$ does not contain a $3 \times\lceil n / 3\rceil$ submatrix consisting entirely of ones. Consider any set of three rows. Then the number of columns in which all three rows have value one is no more than $\lceil n / 3\rceil-1$. Let $T$ be the sum of this quantity, taken over all $\left(\begin{array}{l}n \\ 3\end{array}\right)$ sets of three rows. Then $T \leq(\lceil n / 3\rceil-1)\left(\begin{array}{c}n \\ 3\end{array}\right)$.

For $1 \leq i \leq n$, let $k_{i}$ denote the number of ones in the $i$ th column. Then $m=\sum_{i=1}^{n} k_{i}$ is the number of ones in the entire matrix and $T=\sum_{i=1}^{n}\left(\begin{array}{c}k_{i} \\ 3\end{array}\right)$. By conixity, $T \geq n\left(\begin{array}{c}m / n \\ 3\end{array}\right)$. This implies that $(\lceil n / 3\rceil-1)(n-1)(n-2) \geq$ 
$\frac{m}{n}\left(\frac{m}{n}-1\right)\left(\frac{m}{n}-2\right)$. Let $x=m / n-1$. Then $x^{3}-x-2 u \leq 0$. Since $u^{2}-1 / 27>0$ for $n \geq 4$, the formula for the roots of cubic equations implies that $x \leq$ $\sqrt[3]{u+v}+\sqrt[3]{u-v}$ and, hence, $m \leq n(1+\sqrt[3]{u+v}+\sqrt[3]{u-v})$.

For some small values of $n$, the upper bound on $M(n)$ implied by the following result is better. Like lemma 4, it only uses corollary 1 for $s=2$.

Lemma 6 Suppose $D$ is an $n \times n$ representative matrix, $n \geq 2$. Then $D$ contains at most $K=(n-1)(\lceil 3 n / 2\rceil-2)-(n-2)(\lceil 3 n / 4\rceil-1)+3$ ones.

Proof For $1 \leq i \leq n$, let $k_{i}$ denote the number of ones in the $i$ th row. Without loss of generality, assume $k_{i} \geq k_{i+1}$ for $1 \leq i<n$.

If $k_{1} \leq\lceil 3 n / 4\rceil-2$, then the total number of ones in $D$ is

$$
\sum_{i=1}^{n} k_{i} \leq n(\lceil 3 n / 4\rceil-2) \leq K .
$$

Therefore, assume $k_{1} \geq\lceil 3 n / 4\rceil-1$.

If any row, other than the first, contains $\lceil 3 n / 4\rceil-k_{1}$ ones, then $D$ contains a $2 \times\lceil n / 2\rceil$ submatrix consisting entirely of ones. Thus, by lemma 3 , we may assume that no row, other than the first, contains more than $\lceil 3 n / 2\rceil-k_{1}-1$ ones. Let $s$ be the number of rows which contain exactly this many ones. Then the total number of ones in the matrix is bounded by $k_{1}+s\left(\lceil 3 n / 2\rceil-k_{1}-\right.$ $1)+(n-s-1)\left(\lceil 3 n / 2\rceil-k_{1}-2\right)$ which equals $s-(n-2) k_{1}+(n-1)(\lceil 3 n / 2\rceil-2)$.

The $s$ rows must have ones where row one has zeros. By corollary 1, we must have that $s\left(n-k_{1}\right) \leq n$, so the number of ones in the matrix is bounded by

$$
\begin{aligned}
& \left\lfloor\frac{n}{n-k_{1}}\right\rfloor-(n-2) k_{1}+(n-1)(\lceil 3 n / 2\rceil-2) \\
\leq & 3-(n-2)(\lceil 3 n / 4\rceil-1)+(n-1)(\lceil 3 n / 2\rceil-2) .
\end{aligned}
$$

The following examples show that lemma 4 gives a tight bound for the problem of putting as many ones as possible in a matrix without violating the conditions in corollary 1 , for $n=5$ and $n=8$. Ad hoc arguments show that the second matrix, with 21 ones, has the largest possible number of ones for $n=6$, and the third matrix, with 31 ones, has the largest possible number 
of ones for $n=7$.

$$
\begin{aligned}
& {\left[\begin{array}{lllll}
0 & 1 & 1 & 1 & 1 \\
1 & 1 & 1 & 0 & 0 \\
1 & 1 & 0 & 1 & 0 \\
1 & 0 & 1 & 0 & 1 \\
1 & 0 & 0 & 1 & 1
\end{array}\right]\left[\begin{array}{llllll}
1 & 1 & 1 & 1 & 0 & 0 \\
1 & 1 & 0 & 0 & 1 & 1 \\
0 & 0 & 1 & 1 & 1 & 1 \\
1 & 0 & 1 & 0 & 1 & 0 \\
0 & 1 & 1 & 0 & 0 & 1 \\
1 & 0 & 0 & 1 & 0 & 1
\end{array}\right]\left[\begin{array}{lllllll}
1 & 1 & 1 & 1 & 1 & 0 & 0 \\
1 & 1 & 1 & 0 & 0 & 1 & 1 \\
1 & 0 & 0 & 1 & 1 & 1 & 1 \\
1 & 0 & 1 & 0 & 1 & 0 & 1 \\
0 & 1 & 0 & 1 & 1 & 0 & 1 \\
0 & 0 & 1 & 1 & 0 & 1 & 1 \\
0 & 1 & 1 & 0 & 1 & 1 & 0
\end{array}\right] } \\
& {\left[\begin{array}{llllllll}
1 & 1 & 1 & 1 & 1 & 0 & 0 & 0 \\
1 & 1 & 1 & 0 & 0 & 1 & 1 & 0 \\
1 & 1 & 0 & 1 & 0 & 1 & 0 & 1 \\
1 & 0 & 1 & 1 & 0 & 0 & 1 & 1 \\
0 & 1 & 1 & 0 & 1 & 1 & 0 & 1 \\
1 & 0 & 0 & 0 & 1 & 1 & 1 & 1 \\
0 & 1 & 0 & 1 & 1 & 0 & 1 & 1 \\
0 & 0 & 1 & 1 & 1 & 1 & 1 & 0
\end{array}\right] }
\end{aligned}
$$

It is possible that tighter results could be obtained by considering the $k \times$ $(\lfloor n / k\rfloor+1)$ submatrices for all $k \geq 2$, simultaneously, but this seems to be a hard problem. However, even if we could exactly determine the maximum number of ones that can be in an $n \times n$ matrix that does not contain any $k \times(\lfloor n / k\rfloor+1)$ submatrix consisting only of ones, for all $k \geq 2$, we might still not have tight upper bounds for our original problem. For example, the best known lower bound for $M(5)$ is 12 , though it is possible to put 16 ones in a $5 \times 5$ matrix satisfying the conditions of corollary 1 . It seems that other techniques might be necessary to prove exact bounds.

In the next section, we demonstrate some other techniques which could be useful.

\section{A tight bound for $2 \times 2$ matrices}

In this section, we prove that $M(2) \leq 2$ using different techniques. The example in the introduction shows that this upper bound is tight.

Notice that a function that can be expressed as an affine combination of the variables $x_{1}, x_{2}, \ldots, x_{k}$ over $\operatorname{GF}(2)$ is either the constant 0 or 1 or a parity 
function of a subset of those variables. To prove the upper bound, we first need to develop some properties of the product of two such functions.

Let $f, g:\{0,1\}^{k} \rightarrow\{0,1\}$ be constant or parity functions. Then

$$
f\left(x_{1}, \ldots, x_{k}\right\}=f_{0}+\sum_{i=1}^{k} f_{i} x_{i} \text { and } g\left(x_{1}, \ldots, x_{k}\right)=g_{0}+\sum_{i=1}^{k} g_{i} x_{i}
$$

for some $f_{0}, \ldots, f_{k}, g_{0}, \ldots, g_{k} \in\{0,1\}$. Let $\overrightarrow{0} \in\{0,1\}^{k}$ denote the all zero vector and, for any subset $S \subseteq\{1, \ldots, k\}$, let $\overrightarrow{0}^{(S)} \in\{0,1\}^{k}$ denote the vector such that

$$
\overrightarrow{0}_{i}^{(S)}= \begin{cases}1 & \text { if } i \in S \\ 0 & \text { if } i \notin S .\end{cases}
$$

An assignment of a value in $\{0,1\}^{k}$ to $x_{1}, x_{2}, \ldots, x_{k}$ will be called an input.

Lemma 7 If $f \cdot g=1$, then $f=g=1$.

Proof Suppose $f \cdot g=1$. Since $1=(f \cdot g)(\overrightarrow{0})=f(\overrightarrow{0}) \cdot g(\overrightarrow{0})=f_{0} g_{0}$, it follows that $f_{0}=g_{0}=1$.

Now consider $i \in\{1, \ldots, k\}$. Since $1=(f \cdot g)\left(\overrightarrow{0}^{(\{i\})}\right)=\left(f_{i}+f_{0}\right)\left(g_{i}+g_{0}\right)=$ $\left(f_{i}+1\right)\left(g_{i}+1\right)$, it follows that $f_{i}=g_{i}=0$. Thus $f=g=1$.

Lemma 8 If $f, g$, and $h$ are parity functions and $f \cdot g=h$, then $f=g=h$.

Proof Since $f, g$ and $h$ are parity functions, they are satisfied by (i.e. have value 1 for) exactly half the inputs. But the inputs that satisfy $h$ are the inputs that satisfy both $f$ and $g$. Thus, the inputs that satisfy $h$ are contained in the set of inputs that satisfy $f$, and in the set of inputs that satisfy $g$. Therefore, $f=h$ and $g=h$.

Lemma 9 If $f$ and $f^{\prime}$ are parity functions and $g$ and $g^{\prime}$ are either constant or parity functions such that $f \cdot g+f^{\prime} \cdot g^{\prime}=1$, then

$$
\begin{aligned}
& f=f^{\prime}+1 \\
& g=f \text { or } g=1, \text { and } \\
& g^{\prime}=f^{\prime} \text { or } g^{\prime}=1 .
\end{aligned}
$$

Proof Since $f$ and $f^{\prime}$ are parity functions, they are satisfied by exactly half the inputs. The inputs that satisfy $f \cdot g$ are a subset of those that satisfy $f$; 
thus $f \cdot g$ is satisfied by at most half the inputs. This is also true for $f^{\prime} \cdot g^{\prime}$. But $f \cdot g+f^{\prime} \cdot g^{\prime}=1$, so every input satisfies either $f \cdot g$ or $f^{\prime} \cdot g^{\prime}$. Therefore, $f \cdot g$ and $f^{\prime} \cdot g^{\prime}$ are each satisfied by exactly half the inputs.

For $f \cdot g$ to be satisfied for exactly half the inputs, it must be the case that $g$ is satisfied by all inputs that satisfy f. This implies that $f \cdot g=f$. If $g \neq 1$, then by lemma $8, f=g$. Similarly, $f^{\prime} \cdot g^{\prime}=f^{\prime}$ and either $g^{\prime}=f^{\prime}$ or $g^{\prime}=1$. Hence, $1=f \cdot g+f^{\prime} \cdot g^{\prime}=f+f^{\prime}$, so $f=f^{\prime}+1$.

Theorem $1 M(2) \leq 2$.

Proof Let

$$
A=\left[\begin{array}{ll}
f_{11} & f_{12} \\
f_{21} & f_{22}
\end{array}\right] \text { and } B=\left[\begin{array}{ll}
g_{11} & g_{12} \\
g_{21} & g_{22}
\end{array}\right],
$$

where $f_{11}, f_{12}, f_{21}, f_{22}, g_{11}, g_{12}, g_{21}$, and $g_{22}$ are constant or parity functions of the variables $a_{1}, a_{2}, a_{3}, b_{1}, b_{2}, b_{3}$. Suppose, to obtain a contradiction, that $a_{1} b_{1}, a_{2} b_{2}$, and $a_{3} b_{3}$ are three of the four entries in the product matrix $C=$ $A \cdot B$. Without loss of generality, we may assume that

$$
\begin{aligned}
& f_{11} \cdot g_{11}+f_{12} \cdot g_{21}=a_{1} b_{1} \\
& f_{11} \cdot g_{12}+f_{12} \cdot g_{22}=a_{2} b_{2}, \text { and } \\
& f_{21} \cdot g_{12}+f_{22} \cdot g_{22}=a_{3} b_{3} .
\end{aligned}
$$

Consider the functions $f_{11}^{\prime}, f_{12}^{\prime}, f_{21}^{\prime}, f_{22}^{\prime}, g_{11}^{\prime}, g_{12}^{\prime}, g_{21}^{\prime}$, and $g_{22}^{\prime}$ that result from setting $a_{1}=b_{1}=a_{3}=b_{3}=1$. These functions are also constant or parity functions. Now

$$
\begin{aligned}
& f_{11}^{\prime} \cdot g_{11}^{\prime}+f_{12}^{\prime} \cdot g_{21}^{\prime}=1 \\
& f_{11}^{\prime} \cdot g_{12}^{\prime}+f_{12}^{\prime} \cdot g_{22}^{\prime}=a_{2} b_{2}, \text { and } \\
& f_{21}^{\prime} \cdot g_{12}^{\prime}+f_{22}^{\prime} \cdot g_{22}^{\prime}=1 .
\end{aligned}
$$

If $f_{11}^{\prime}=0$, then $f_{12}^{\prime} \cdot g_{21}^{\prime}=1$; so by lemma $7, f_{12}^{\prime}=g_{21}^{\prime}=1$. This implies $a_{2} b_{2}=g_{22}^{\prime}$, which is impossible, since $a_{2} b_{2}$ is neither a constant nor a parity function. Thus $f_{11}^{\prime} \neq 0$. Similarly, $f_{12}^{\prime}, g_{12}^{\prime}, g_{22}^{\prime} \neq 0$.

If $f_{11}^{\prime}, f_{12}^{\prime} \neq 1$, then, by lemma $9, f_{12}^{\prime}=f_{11}^{\prime}+1$. Similarly, if $g_{12}^{\prime}, g_{22}^{\prime} \neq 1$, then $g_{22}^{\prime}=g_{12}^{\prime}+1$. If both these equations are true, then

$$
\begin{aligned}
a_{2} b_{2} & =f_{11}^{\prime} \cdot g_{12}^{\prime}+f_{12}^{\prime} \cdot g_{22}^{\prime} \\
& =f_{11}^{\prime} \cdot g_{12}^{\prime}+\left(f_{11}^{\prime}+1\right) \cdot\left(g_{12}^{\prime}+1\right) \\
& =1+f_{11}^{\prime}+g_{12}^{\prime} .
\end{aligned}
$$


This is impossible, since $1+f_{11}^{\prime}+g_{12}^{\prime}$ is a constant or parity function. Therefore, at least one of $f_{11}^{\prime}, f_{12}^{\prime}, g_{12}^{\prime}$, and $g_{22}^{\prime}$ is 1 . Without loss of generality, say $f_{11}^{\prime}=1$. Then $a_{2} b_{2}=g_{12}^{\prime}+f_{12}^{\prime} \cdot g_{22}^{\prime}$.

Now either $g_{12}^{\prime}=1$ or $g_{22}^{\prime}=1$. Otherwise, by lemma $9, a_{2} b_{2}=g_{12}^{\prime}+f_{12}^{\prime}$. $\left(g_{12}^{\prime}+1\right)$ or, equivalently, $a_{2} b_{2}+\left(f_{12}^{\prime}+1\right) \cdot\left(g_{12}^{\prime}+1\right)=1$. But this would contradict lemma 9 , since $a_{2}, b_{2}, g_{12}^{\prime}+1 \neq 0,1$ and $b_{2} \neq a_{2}$.

Furthermore, $g_{22}^{\prime} \neq 1$ or else $a_{2} b_{2}=g_{12}^{\prime}+f_{12}^{\prime}$. This is impossible because $g_{12}^{\prime}+f_{12}^{\prime}$ is a constant or parity function. Therefore, $g_{12}^{\prime}=1$.

Then $a_{2} b_{2}=1+f_{12}^{\prime} \cdot g_{22}^{\prime}$ or, equivalently, $a_{2} b_{2}+f_{12}^{\prime} \cdot g_{22}^{\prime}=1$. Since $a_{2} \neq 0,1$ and $b_{2} \neq 1, a_{2}$, it follows from lemma 9 and the commutativity of $f_{12}^{\prime}$ and $g_{22}^{\prime}$, that neither $f_{12}^{\prime}$ nor $g_{22}^{\prime}$ can be parity functions; thus, they are constant. But this implies that $a_{2} b_{2}$ is also constant, which it is not. Hence $M(2) \leq 2$.

\section{Conclusion}

The following theorem summarizes the results of sections 2 and 3 .

Theorem 2 Let $u=\frac{1}{2}(\lceil n / 3\rceil-1)(n-l)(n-2)$ and $v=\sqrt{u^{2}-1 / 27}$. Then for $n \geq 4$,

$$
M(n) \leq n(1+\sqrt[3]{u+v}+\sqrt[3]{u-v})=n^{2} / \sqrt[3]{3}+O(n)
$$

In addition, $M(2)=2, M(3)=6$, and $M(4)=9$.

The above theorem states the best asymptotic results, but for some small values of $n$, lemmas 4 and 6 give better results, as the following table shows. The theorem gives the best results for larger values of $n$ than those shown in the table. 


\begin{tabular}{|l||r|r|r|r|r|r|r|r|r|r|}
\hline $\mathrm{n}$ & 1 & 2 & 3 & 4 & 5 & 6 & 7 & 8 & 9 & 10 \\
\hline \hline Lemma 4 & - & 2 & 6 & 9 & 16 & 22 & 33 & 40 & 55 & 65 \\
Lemma 5 & - & - & - & 12 & 17 & 23 & 35 & 43 & 53 & 70 \\
Lemma 6 & - & 4 & 7 & 11 & 18 & 22 & 32 & 43 & 57 & 64 \\
\hline
\end{tabular}

\begin{tabular}{|l||r|r|r|r|r|r|r|r|r|r|}
\hline $\mathrm{n}$ & 11 & 12 & 13 & 14 & 15 & 16 & 17 & 18 & 19 & 20 \\
\hline \hline Lemma 4 & 83 & 95 & 117 & 130 & 156 & 172 & 201 & 219 & 251 & 271 \\
Lemma 5 & 82 & 95 & 118 & 134 & 150 & 179 & 198 & 217 & 252 & 274 \\
Lemma 6 & 81 & 99 & 120 & 130 & 154 & 179 & 207 & 220 & 251 & 283 \\
\hline
\end{tabular}

\begin{tabular}{|l||r|r|r|r|r|r|r|r|r|r|}
\hline $\mathrm{n}$ & 21 & 22 & 23 & 24 & 25 & 26 & 27 & 28 & 29 & 30 \\
\hline \hline Lemma 4 & 307 & 330 & 369 & 393 & 436 & 463 & 510 & 538 & 588 & 619 \\
Lemma 5 & 297 & 337 & 363 & 390 & 435 & 465 & 495 & 546 & 579 & 612 \\
Lemma 6 & 318 & 334 & 372 & 411 & 453 & 472 & 517 & 563 & 612 & 634 \\
\hline
\end{tabular}

\begin{tabular}{|l||r|r|r|r|r|r|r|r|r|r|}
\hline $\mathrm{n}$ & 31 & 32 & 33 & 34 & 35 & 36 & 37 & 38 & 39 & 40 \\
\hline \hline Lemma 4 & 673 & 706 & 763 & 798 & 859 & 896 & 960 & 999 & 1067 & 1109 \\
Lemma 5 & 669 & 705 & 742 & 804 & 844 & 884 & 952 & 995 & 1039 & 1112 \\
Lemma 6 & 686 & 739 & 795 & 820 & 879 & 939 & 1002 & 1030 & 1096 & 1163 \\
\hline
\end{tabular}

\begin{tabular}{|l||r|r|r|r|r|r|r|r|r|r|}
\hline $\mathrm{n}$ & 41 & 42 & 43 & 44 & 45 & 46 & 47 & 48 & 49 & 50 \\
\hline \hline Lemma 4 & 1180 & 1223 & 1298 & 1344 & 1422 & 1470 & 1552 & 1602 & 1687 & 1739 \\
Lemma 5 & 1159 & 1207 & 1285 & 1335 & 1386 & 1471 & 1524 & 1579 & 1668 & 1726 \\
Lemma 6 & 1233 & 1264 & 1337 & 1411 & 1488 & 1522 & 1602 & 1683 & 1767 & 1804 \\
\hline
\end{tabular}

\section{Acknowledgements}

We are grateful to Gudmund Frandsen for helpful discussions. We would also like to thank Richard Anstee, Jørgen Brandt, Cary Huffman, and Richard Nowakowski.

\section{References}

[BBP91] J. Boyar, G. Brassard, and R. Peralta. Subquadratic zeroknowledge. In Proceedings of the 32nd IEEE Symposium on Foundations 
of Computer Science, pages 69-78, 1991.

[GZ91] R.K. Guy and S. Znám. A problem of Zarankiewicz. In W.T. Tutte, editor, Recent Progress in Combinatorics: Proceedings of the Third Waterloo Conference on Combinatorics, May 1968, pages 237-243, 1991.

[HC58] C. Hyltén-Cavallius. On a combinatorical problem. Colloq. Math., pages 59-65, 1958.

[KST54] T. Kövari, V.T. Sós, and P. Turán. On a problem of K. Zarankiewicz. Colloq. Math., pages 50-57, 1954.

[Rom75] S. Roman. A problem of Zarankiewicz. Journal of Combinatorial Theory (A), pages 187-198, 1975.

[SS42] R. Salem and D. C. Spencer. On sets of integers which contain no three terms in arithmetical progression. In Proceedings of the National Academy of Sciences of the United States of America, pages 561-563, 1942.

[Sze] E. Szemerédi. Personal communication through G. Brassard, S. Kannan, S. Rudich and G. Tardos.

[Win70] S. Winograd. On the number of multiplications necessary to compute certain functions. Comm. on Pure and Applied Mathematics, pages 165-179, 1970.

[Zar51] K. Zarankiewicz. Problem P 101. Colloq. Math., page 301, 1951. 